\title{
Percepción de la violencia y el capital social en Nuevo León, México
}

\author{
María de Jesús Ávila Sánchez ${ }^{1}$ (iD \\ Eric Omar Pantoja Donia² \\ José Alfredo Jáuregui Díaz ${ }^{3}$
}

\begin{abstract}
El presente artículo tiene como objetivo analizar la relación entre algunas dimensiones del capital social y la incidencia observacional de diferentes tipos de violencia en la Zona Metropolitana de Monterrey. En este análisis se utilizó como fuente primaria de información la base de datos de la Encuesta de Cohesión Social para la Prevención de la Violencia y la Delincuencia (Ecopred). Los datos de la encuesta fueron analizados aplicando la técnica estadística de componentes principales para determinar la tipología del capital social y de los diferentes tipos de violencia. Posteriormente se elaboró un modelo de regresión lineal múltiple. A partir de los resultados se demostró que tres dimensiones del capital social, las redes sociales organizadas, la confianza interpersonal fuerte y las altas expectativas sociales tuvieron un efecto positivo en la disminución de la violencia por convivencia, pandillas, contra el patrimonio y por delitos menores. En conclusión, poseer alguna dimensión de capital social es una estrategia comunitaria para contener la violencia.

Palabras clave: capital social; confianza; violencia; delitos; Nuevo León
\end{abstract}

\section{Introducción}

En México se experimentó un aumento notable de la inseguridad y la violencia, como resultado de la lucha frontal del gobierno mexicano contra el narcotráfico durante los últimos 17 años. La Zona Metropolitana de Monterrey (ZMM) no fue la excepción. Los reportes de casos vinculados con el crimen organizado (secuestros, robo con violencia, asesinatos, balaceras en la vía pública) tuvieron un repunte en el año 2010, situación que continúa. Sólo entre 2015 y 2016 la incidencia delictiva del fuero común muestra que los homicidios dolosos se incrementaron $43 \%$, los robos $31 \%$, los secuestros $29 \%$ y los casos de violación sexual 10\% (SESNSP, 2017).

\footnotetext{
1 Universidad Autónoma de Nuevo León, Instituto de Investigaciones Sociales. Monterrey (NL), México. E-mail: <marycolef@yahoo.com>.

2 Universidad Autónoma de Nuevo León, Instituto de Investigaciones Sociales. Monterrey (NL), México. E-mail: <pantoja.eric@gmail.com>.

3 Universidad Autónoma de Nuevo León, Instituto de Investigaciones Sociales. Monterrey (NL), México.

E-mail: <alfjadi@yahoo.com.mx>.
} 
Ante este clima de inseguridad y violencia, una de las medidas que tomaron las personas en la ZMM para el cuidado de la propiedad privada y la integridad personal fue la conformación de grupos vecinales de vigilancia. Diversos estudios han señalado los beneficios de las asociaciones vecinales como generadores de nuevas formas de participación autónomas donde se crea un vínculo más estrecho de confianza y comunicación con los vecinos (Safa y Ramírez, 2011).

Los grupos de vigilancia surgen de la suma y la superposición de las redes de relaciones vecinales y experiencias de las personas, construidas diariamente basadas en la reciprocidad, cooperación y confianza entendidas como capital social. Estas redes relacionales pueden representar un recurso importante para individuos y grupos sociales para resolver problemas colectivos, al establecer prácticas de cooperación e intercambios recíprocos capaces de reducir los costos de transacción y ampliar la cohesión socioeconómica y confianza entre actores locales (Bellanca y Brandt, 2010).

Hirschman (1984) sostiene que el capital social es el único recurso que no disminuye o pierde valor con el uso; en cambio, tiene el potencial de aumentar continuamente, a menudo de manera intangible. Para Lunecke y Ruiz (2006), el capital social actúa como una red de seguridad y favorece la resolución pacífica de conflictos al interior de las comunidades. En tanto que para Portela y Neira (2012) tiene una influencia en el bienestar subjetivo y la felicidad referente a los aspectos individuales y materiales de la vida, por lo que podríamos esperar que personas y comunidades con altos niveles de capital social viven en espacios menos violentos.

El objetivo del presente artículo es analizar la relación entre algunos componentes del capital social (como concentración o extensión de las relaciones sociales, la confianza interpersonal basada en la reciprocidad particular y la confianza estructural) y la incidencia observacional de diferentes manifestaciones de la violencia en la Zona Metropolitana de Monterrey. Se parte del supuesto que no existe una relación univoca entre el capital social y la violencia como ha sido demostrado en la literatura, sino que algunos de los componentes del capital social son los que pudieran tener una mayor o menor influencia en la incidencia de algunas de las manifestaciones de la violencia como guía en el desarrollo de la investigación. Es decir, algunos componentes del capital social pudieran tener un efecto positivo al inhibir o contener algunas formas de violencia específicas.

En su realización se utilizó como fuente primaria de información la base de datos de la Encuesta de Cohesión Social para la Prevención de la Violencia y la Delincuencia (Ecopred). Los datos de la encuesta fueron analizados aplicando la técnica estadística de componentes principales para determinar la tipología del capital social y de los diferentes tipos de violencia. Posteriormente se elaboró un modelo de regresión lineal múltiple.

Este artículo se divide en cinco apartados, en el primero se explica el término capital social y sus dimensiones utilizadas en el estudio; en el segundo se define violencia; en el tercero se muestra cómo se relaciona el capital social con la violencia. A continuación, en el cuarto apartado se presentan los métodos utilizados para la recopilación y análisis de 
los datos; en el quinto apartado se presentan los resultados encontrados y la discusión de los mismos. Finalmente, en el sexto apartado se discuten las conclusiones más sobresalientes del estudio sobre la importancia del capital social en la inhibición de las violencias en la ZMM.

\section{El capital social y dimensiones utilizadas en el estudio}

Definir el término capital social fue fundamental para la realización de esta investigación así como dos de sus componentes, las redes sociales y la confianza, las cuales servirán como guías en el desarrollo de la misma -tarea que se realiza desde una visión sociológica.

¿Qué es el capital social? Frecuentemente se ubica el origen del concepto de capital social en la idea de la cohesión social propuesta por Émile Durkheim, referida a la existencia de relaciones y lazos sociales fuertes que permiten mantener la unión entre los individuos y evitar el conflicto en una sociedad. De acuerdo con Durkheim (1985), la cohesión social es producida por la existencia de un imaginario social compartido compuesto por un sistema de normas, valores, sentimientos e ideas comunes entre todas las personas que integran la sociedad y por la presencia de lazos sociales de parentesco e impersonales que surgen de la necesidad de cooperación entre los individuos para atender las tareas de reproducción social.

Son Bourdieu, Coleman y Putnam quienes introducen explícitamente el concepto de capital social en la segunda mitad de la década de 1970, aunque existen importantes diferencias entre los tres autores. En términos generales, el capital social está relacionado con el valor de la confianza y con las relaciones sociales (Rosero-Bixby, 2006). Para Bourdieu y Wacquant (1992), el capital social es "la suma de los recursos existentes o potenciales, de la que se hace acreedor un individuo o grupo en virtud de poseer una red perdurable de relaciones más o menos institucionalizadas de mutua familiaridad y reconocimiento" (p. 178). En otras palabras, pertenencia a un grupo que proporciona a cada uno de sus miembros el apoyo del capital construido colectivamente.

La cantidad de capital social a disposición de las personas depende de la extensión de la red de vínculos sociales que se pueden movilizar y la cantidad de recursos financieros y culturales que pueden reunir los participantes de la red. Por lo tanto, el capital social es creado y acumulado en las relaciones sociales, además de que puede ser utilizado por las personas como un recurso (Bourdieu, 1985). El capital social tiene un doble objetivo, facilitar la cooperación entre el individuo y la red social en la creación de vínculos de confianza y movilizar recursos financieros, humanos, culturales y políticos (Faist, 2004). En este sentido, Coleman (1988) señala que "el capital social facilita las acciones y la satisfacción de los intereses de los actores sociales que no podrían haberse obtenido en su ausencia o que podrían haberse logrado sólo a un costo mayor" (p. 98). 
Para Ostrom y Ahn (2003) el capital social no sólo reporta beneficios individuales sino colectivos, por lo que puede ser considerado un bien común, en la medida en que la pertenencia a un colectivo incrementa los beneficios de los miembros, incluso de aquellos que no contribuyeron a la consolidación de ese bien. Por ejemplo, un vecindario donde los vecinos se organizan para crear un grupo de vigilancia para aumentar la seguridad local, beneficiando a todos los vecinos incluso a aquellos que prefieren ahorrarse las molestias de contribuir al cuidado de la propiedad de los demás (Cruz y Contreras-Ibáñez, 2015, p. 141).

Existen diversas propuestas de autores que acotan el capital social en cinco dimensiones:

i. Lazos y redes sociales (Bourdieu, 1985; Bourdieu y Wacquant, 1992; Lin, 2001; Faist, 2004)

ii. Confianza (Luhmann, 1996; Coleman, 1990)

iii. Normas y reglas de acción colectiva (Coleman, 1988)

iv. Asociativismo (Putnam, 1993; Narayan y Pritchett, 1999)

v. Empoderamiento (Grootaert et al., 2004)

\section{Dimensiones del capital social utilizadas en la investigación}

En este artículo, el capital social será abordado desde dos dimensiones: una a nivel micro que se refiere a las redes sociales -interacción entre los individuos quienes son tanto actores como beneficiarios e inversionistas de los recursos que emanan del capital social según lo planteado por Bourdieu (1985)- y otra a nivel meso basado en la confianza estructural propuesta por Coleman (1990). A continuación nos centraremos en los recursos que poseen los integrantes de una red y los factores estructurales que facilitan su movilización (Lin, 2001).

- Dimensión micro - redes sociales

Las redes sociales, que están conformadas por diferentes tipos de lazos sociales entre los individuos, se refieren a las redes horizontales que surgen de las relaciones entre los miembros de un grupo con un mismo proyecto y recurso de poder dentro de un campo. Por ejemplo, aquellos lazos que se establecen entre vecinos, parientes y amigos con la intención de intercambiar favores, bienes y servicios estratégicos para el desarrollo de la vida cotidiana de los miembros, grupos o familias. Tales lazos generan una reciprocidad particular en las que se espera que el otro retribuya las atenciones proporcionadas en un momento determinado.

- Dimensión meso - la confianza

Entender por qué las personas confían y cómo se cultiva la confianza en las relaciones sociales ha sido objeto de diversas disciplinas, pero no existe un consenso sobre el enfoque para definirla y operacionalizarla. En esta investigación se abordan dos tipos de 
confianza: la interpersonal basada en la reciprocidad particular y la confianza estructural que Coleman (1988) denomina confianza en el ámbito social.

a) La confianza interpersonal

Se entiende como una actitud basada en las expectativas del comportamiento de otra persona que participa en una relación fundamentada en el afecto que existe entre ambos (Durston, 2000), por lo que constituye el resultado de la interacción previa o derivada de la membresía en un mismo grupo social o en una relación diádica (Foster, apud Duston, 2000).

De acuerdo con la antropología, la confianza se basa en dos elementos. El primero es el principio de reciprocidad, que le da una base cultural, y el segundo es lo emocional, que se expresa en el afecto que las personas tienen hacia quienes confían en ellas (Durston, 2000). La confianza significa superar la aversión al riesgo, por lo menos a esas personas o grupos, que de acuerdo con la experiencia se espera que respondan con reciprocidad a un acto, por ejemplo, de generosidad.

Confiar implica ceder el control de bienes propios al otro, que pueden ser vecinos, parientes y amigos. Los bienes pueden ser de diversa naturaleza, pero se destacan los préstamos de herramientas, dinero o comida y ayuda para acceder a diversos servicios. Es necesario que la confianza interpersonal esté inmersa en reciprocidad particular, basada en un entendimiento informal e implícito entre dos personas que mantienen intercambios en el tiempo, de cosas y actos del mismo valor, que se apoyan en la confianza mutua.

Esto genera una forma de capital acumulado de reciprocidad particular para que una persona pueda reclamar a otras personas, a quienes les ha realizado algún favor o prestamos en el pasado, en un momento de necesidad. Según Lomnitz (1978) es a partir de la movilización de estos recursos que los grupos son capaces de generar estrategias para enfrentar las necesidades y amenazas de la vida cotidiana aún en contextos de vulnerabilidad económica y laboral.

La relación entre confianza interpersonal y reciprocidad particular se refuerza de tal manera, que se esperaría que cuanto mayor sea el valor material o simbólico del bien entregado o compartido mayor será el grado de confianza interpersonal depositada en la otra persona y la expectativa de reciprocidad particular.

b) Confianza estructural

Es propia de un orden social complejo en el que se tiende a perder la familiaridad, y la refuerzan mecanismos como la ley, es decir, como un elemento estructural al que Coleman (1988) denomina confianza en el ámbito social. Lo que, por ejemplo, permite a las personas pasear por las noches por las calles de una gran ciudad sin temer por su seguridad, es decir, con confianza. Se basa en representaciones y valores compartidos como condiciones de posibilidad del capital social. Debido a que la ausencia de confianza estructural obstaculiza la existencia de las redes sociales, tanto primarias (familiares, 
amistad, etc.) como organizadas (organizaciones vecinales), dificulta la construcción de ámbitos de pertenencia colectiva y de proyectos de vida personal significativos.

Una forma de aproximarse a esta confianza estructural es a través de las expectativas de vida futura de los individuos, las cuales son resultado de un sistema compartido de normas y valores que propician que los miembros de una comunidad o una localidad actúen de manera colectiva en la persecución de un fin común. De este modo, el capital social tiene como base la incorporación del bienestar de los otros en el bienestar propio, de manera que cuando se lleva a cabo una acción colectiva para generar un bien común se aprovecha la buena disposición de cada individuo.

La relevancia del capital social de la confianza estructural radica en su capacidad de contribuir al bienestar de las comunidades, por lo que se considera un elemento clave para reducir el riesgo y la incertidumbre (Arriagada, 2003) y, por lo tanto, la percepción de violencia.

\section{¿Qué es la violencia?}

No existe una definición univoca de la violencia, al tratarse de un término polisémico, ambiguo y cruzado por cuestiones políticas (Del Olmo, 2000). Por esa razón, en esta investigación nos referimos a las violencias en plural, dando un margen más amplio en la comprensión de los diferentes tipos de violencia a los que se encuentran expuestos los ciudadanos de las grandes urbes, donde se enfrentan a una multiplicidad de violencias. Esto permite examinar su complejidad y destacar las características más precisas de la forma en que se presentan y las dinámicas que asumen con las dimensiones del capital social.

Dammert y Zúñiga (2007) mencionan que nos encontramos frente a una multiplicidad de violencias las cuales pueden agruparse de acuerdo con su objetivo, espacio geográfico, el individuo o el grupo que ejerce la violencia o quién sufre de ella. En esta investigación nos enfocaremos en dos concepciones de la violencia urbana que según Carreón (1994) se encuentran claramente diferenciadas y estrechamente interrelacionadas: la inseguridad y la criminalidad.

La inseguridad hace referencia a los hechos concretos de violencia objetiva producidos o, lo que es lo mismo, la falta de seguridad, y la percepción de inseguridad que hace relación a la sensación de temor y que tiene que ver con el ámbito subjetivo de la construcción social del miedo generado por la violencia directa o indirecta. En tanto que la criminalidad y el delito son términos que describen a la violencia urbana, debido a que la violencia ejercida en contra de una persona implica siempre la intención de perpetrar un daño contra otro, por lo que contempla una dimensión delictiva y, por tanto, está penada socialmente. En este sentido, la violencia urbana y la criminalidad se encuentran estrechamente vinculadas debido a las relaciones cotidianas inmersas en la dinámica de las comunidades pertenecientes a las zonas urbanas o a las periferias de estas grandes 
zonas, donde casi siempre el resultado del ejercicio de la violencia urbana será un hecho delictivo criminal.

El término de violencia urbana hace referencia al crimen cometido en los entornos públicos de las grandes ciudades. Así, "la violencia urbana es aquella ejercida en el marco de las relaciones y dinámicas mediadas por la convivencia urbana, cuyas expresiones más frecuentes son el robo a mano armada, amenazas, agresiones, golpes, secuestros y homicidio" (Cruz, 1999, p. 260). Se encuentra relacionada con el aumento de la exclusión social, política y económica que experimenta gran parte de la población (Tedesco, 2009), además de la debilidad institucional de los Estados y con la baja calidad de la democracia.

\section{Relación entre capital social y violencia}

Existen diversos estudios que han examinado la relación entre capital social y la violencia. Por ejemplo, Jara (2015) menciona la forma en que el capital social tiene una relación negativa con los niveles de delitos violentos, una vez controladas las variables de densidad poblacional, movilidad de las personas y el hacinamiento. Asimismo, encuentra que es una variable explicativa con una relación positiva si la variable dependiente es homicidios, pero negativa cuando la variable dependiente es robo con violencia.

Sobre las relaciones positivas del capital social y la violencia urbana, Latorre (2004) analizó la influencia de la violencia en la decisión de cada agente de invertir en capital social y la atribución del capital social sobre la decisión que enfrenta el agente violento al atacar cierta localidad. En específico, encuentra que la promoción de capital social constituye una amenaza para el fomento de la violencia y un complemento a las acciones de los organismos gubernamentales sobre los problemas de la localidad. En otras palabras, es una condición necesaria pero no suficiente para solucionar los problemas ocasionados por la violencia.

La inversión en capital social que realizan las personas de la comunidad está influida por las características de la violencia que se observa en su localidad. El resultado de la inversión se dará en la medida en que se acumule suficiente capital social entre los individuos, se fortalezca la cohesión para hacer efectiva su acción, y que las entidades legales y de seguridad apoyen y refuercen el desarrollo de este capital (Latorre, 2004).

La participación ciudadana, el capital social y el asociativismo tienden a crecer a medida que los delitos aumentan en una comunidad, siendo este tipo de organización social un factor de contención que ayuda en la reducción de robos con violencia en las viviendas, violencia intrafamiliar y lesiones, en el espacio comunitario (Latorre, 2004).

En tanto, mediante el análisis de la relación entre violencia y capital social en barrios urbanos críticos en el entorno específico chileno, Lunecke y Ruiz (2006) concluyen que los lazos y vínculos sociales presentes en las comunidades críticas se encuentran desgastados o erosionados debido a la intervención del narcotráfico, el consumo de drogas y la violencia. Cuando las comunidades son etiquetadas con denominaciones negativas, se 
generan estereotipos que repercuten en la exclusión social, generando una pérdida del recurso acumulado por sus habitantes durante décadas de organización, trabajo comunitario de base y uso extensivo de redes fuertes y débiles. Estos elementos, componentes centrales del capital social, se han visto disminuidos desde los años noventa debido, en gran parte, al lado oscuro del capital social donde se encuentran cimentadas las relaciones asociativas del narcotráfico.

La relación entre las dimensiones del capital social con los distintos tipos de delitos muestra que la cantidad de interacciones sociales es más alta en delitos menores (como hurto y robo de automóviles), moderada en delitos más graves (asalto y robo) y casi insignificante en homicidios y violaciones (Glaeser, Sacerdote y Scheinkman, 1996). Pero una medida combinada de control social informal, cohesión y confianza es un fuerte predictor de menores tasas de violencia después de tener en cuenta la composición del vecindario y los efectos recíprocos de la violencia (Sampson, Raudenbush y Earls, 1997).

López-Rodríguez, Soloaga y De la Torre (2014) demuestran que el nivel de asociativismo disminuye la percepción de violencia en México. En tanto que la disminución del capital social en América Latina incide en mayores tasas de delincuencia en las ciudades (Rosero-Bixby, 2006). Aunque para autores como Rosenfeld, Messner y Baumer (2001), la relación es contraria, la confianza social y el compromiso cívico tienen efectos negativos en la delincuencia y la violencia.

En resumen, no existe un consenso claro en cuanto a la relación entre capital social y violencia, sin embargo algunos estudios han demostrado que las altas tasas de violencia están significativamente asociadas a un menor capital social. Igualmente, diversos estudios han evidenciado los efectos negativos de la violencia en el capital social que se manifiesta en un aumento de los conflictos sociales y el debilitamiento de los vínculos familiares y comunitarios (Portes, 1995 y Lunecke y Ruiz, 2006). Los estudios realizados sobre la relación entre capital social y violencia no son concluyentes y no proporcionan una base suficiente para explicar con claridad la forma en que los mecanismos específicos del capital social influyen en las diferentes manifestaciones de la violencia y en cuáles los efectos son más importantes.

En este sentido surgen dos grandes preguntas: ¿Las dimensiones constitutivas del capital social, las relaciones sociales, la confianza interpersonal basada en la reciprocidad y la confianza estructural, ejercen efectos comparables sobre la incidencia de los diferentes tipos de violencia? ¿Son más relevantes los aspectos particulares del capital social para explicar ciertas formas seleccionadas de violencia (delitos de alto impacto) en comparación con otras formas (por ejemplo, delitos menores)? Una comprensión completa de los procesos que vinculan el capital social con las violencias requiere que las respuestas tentativas a tales preguntas sean planteadas y probadas con datos generados en espacios específicos. 


\section{Metodología}

En esta investigación se utilizaron los datos de la Ecopred del año 2014, la cual fue elaborada, aplicada y validada por el Instituto Nacional de Estadística y Geografía (Inegi) en el marco del Subsistema Nacional de Información de Gobierno, Seguridad Pública e Impartición de Justicia (SNIGSPIJ) y del Programa Nacional para la Prevención Social de la Violencia y la Delincuencia. La encuesta tiene entre sus objetivos medir las características sobre variables asociadas a la calidad de la interacción entre los vecinos de la vivienda, así como la confianza que se tienen entre sí. Además, indaga el grado de satisfacción que sienten las personas con respecto a sus viviendas, colonias y/o barrios, así como los patrones de conducta antisocial que existen en su interior y mide características sobre variables asociadas a actividades e intereses que favorezcan la cohesión social, la participación ciudadana e incentiven la prevención de la violencia y el delito.

Fue entrevistado(a) el/la jefe(a) de los hogares en las viviendas particulares habitadas seleccionadas y una población de jóvenes, de 12 a 29 años, que no hayan sido declarado(a) jefe(a) o cónyuge de este(a). La Ecopred proporciona datos a nivel nacional y de zonas metropolitanas y tiene una cobertura geográfica que incluye a 47 ciudades de México. El diseño de muestra fue probabilístico, estratificado, uniétapico y por conglomerados, estos últimos se consideran unidades primarias de muestreo (UPM), pues es en ellos donde se seleccionan en una segunda etapa las viviendas que integran las muestras de la encuesta. El tamaño de muestra fue de 97,850 viviendas. El periodo de levantamiento fue del 6 de octubre al 9 de diciembre de 2014 (Inegi, 2014).

De acuerdo con el objetivo de este artículo se seleccionaron los datos de la Zona Metropolitana de Monterrey, teniendo como unidad de análisis a los(las) jefes(as) de los hogares, ya que se consideró que podrían ser informantes más enterados sobre los temas de seguridad en la comunidad. El tamaño de la muestra de la ZMM fue de 6,824 jefes(as) de hogar, que representan de manera ponderada a 1,099,518 jefes(as). La edad promedio fue de 39.6 años, con una desviación estándar de 21.4 años. El 54.6\% son hombres y el $45.4 \%$ son mujeres. El 52.6\% declararon que realiza una actividad remunerada.

Para aproximarnos a la dimensión de la incidencia de las violencias se seleccionó la pregunta 4.18 del cuestionario de la Ecopred (Figura 1) que indaga sobre la observación de una serie de delitos en la colonia o barrio en el que vive el/la jefe(a) de hogar entrevistado(a). 
Figura 1

Pregunta sobre incidencia de la violencia en la Ecopred

\begin{tabular}{|c|c|}
\hline $\begin{array}{l}\text { 4.18 En lo que va del año, ¿qué tan frecuente ha } \\
\text { observado gente en su colonia o barrio... } \\
\text { REGISTRE EL CODIGO QUE CORRESPONDA A CADA OPCIÓN } \\
\text { Muy frecuente (1) } \\
\text { Frecuente (2) } \\
\text { Poco frecuente (3) } \\
\text { Nunca (4) } \\
\text { No sabe/no responde (9) }\end{array}$ & $\begin{array}{l}\text { 4.19 Ante la situación } \\
\text { (RESPUESTA 4.18) } \\
\text { los vecinos o colonos les han } \\
\text { llamado la atención (1) } \\
\text { los vecinos o colonos se han } \\
\text { organizado para resolverlo (2) } \\
\text { la policía ha intervenido (3) } \\
\text { no se ha hecho nada (4) } \\
\text { No sabe/no responde (9) }\end{array}$ \\
\hline 1 CON CODIGO 4 Y 9 APLICAR PREGUNTA 4.19 & \\
\hline $\begin{array}{l}\text { 01. haciendo ruido (música a alto volumen, fiesta, reparando } \\
\text { algo o realizando alguna actividad doméstica)? [ ] }\end{array}$ & [ ] \\
\hline 02. grafiteando paredes o rayando autos? [ ] & [ ] \\
\hline $\begin{array}{l}\text { 03. rompiendo ventanas de casas, negocios, autos u otros } \\
\text { objetos? [ ] }\end{array}$ & {$[\quad]$} \\
\hline 04. jugando arrancones? [ ] & [ ] \\
\hline 05. tomando alcohol en la calle? [ ] & [ ] \\
\hline 06. vendiendo productos piratas? [ ] & [ ] \\
\hline 07. vendiendo drogas? [ ] & [ ] \\
\hline 08. consumiendo drogas? [ ] & [ ] $]$ \\
\hline 09. bloqueando la calle? [ ] & [ ] \\
\hline 10. peleando entre pandillas? [ ] & [ ] \\
\hline 11. discutiendo o peleando entre vecinos? [ ] & [ ] \\
\hline 12. prostituyéndose? [ ] & [ ] \\
\hline 13. asaltando casas, negocios o vehículos? [ ] & {$[\quad]$} \\
\hline 14. asaltando o robando personas en la calle? [ ] & [ ] \\
\hline 15. amenazando o extorsionando? [ ] & [ ] \\
\hline 16. disparando algún tipo de arma de fuego? [ ] & [ ] \\
\hline
\end{tabular}

Fuente: Inegi (2014).

Debido a la naturaleza de la pregunta seleccionada se decidió usar el término de la incidencia de algún delito. La percepción de inseguridad no tiene necesariamente una correlación directa con los niveles de victimización real, esto debido a que son un conjunto de factores sistémicos propios de la dinámica social los que determinan las características de los imaginarios de la inseguridad y, por lo tanto, de la incidencia de las violencias (Córdova, 2007).

Debido a que la Ecopred no incluye preguntas sobre las cinco dimensiones que han sido examinadas en la literatura sobre capital social, en este artículo se decidió analizar 
sólo dos dimensiones. La primera, a nivel micro, que son los tipos de redes sociales y la confianza interpersonal basada en la reciprocidad particular. $Y$, la segunda, a nivel meso, que se refiere a la confianza estructural basada en las expectativas de vida, a través de 36 preguntas.

Para la dimensión de las redes sociales se seleccionaron veinte preguntas que se transformaron en seis variables:

- Número de familiares.

- Amigos o conocidos que viven cerca.

- Frecuencia con la que se encuentra con ellos en diferentes lugares (las calles de la colonia, la tienda, el mercado, supermercado o centro comercial; los parques, jardines o plazas públicas y en restaurantes, cafés o bares).

- Intensidad de la interacción social con los vecinos (se identifican físicamente; se saludan cada vez que se encuentran; cuando se encuentran, además, se detienen a platicar; en caso de que platiquen, se cuentan los problemas que están enfrentando; se visitan en sus respectivas casas).

- Participación en actividades comunes (para organizar eventos religiosos; fiestas; la seguridad de la colonia; solucionar problemas de servicios públicos; solicitar servicios de la delegación o municipio; para convivir casualmente en un área común o público).

- Membresía del/de la jefe(a) del hogar o la de otro miembro del hogar en alguna organización vecinal.

Para definir el grado de confianza interpersonal basado en la reciprocidad se seleccionaron diez preguntas que reflejan diferentes aspectos del tipo de apoyo social que intercambian con sus vecinos y que miden los diferentes niveles de confianza y expectativas de comportamiento basado en la confianza interpersonal, según el valor del apoyo que creen que les pueden brindar sus vecinos, que van de mayor a menor confianza:

- Les dejaría a los vecinos las llaves de la casa

- Los vecinos les han cuidado a sus hijos

- Cuidarían la casa; recurriría a sus vecinos en caso de un problema

- Prestaría una herramienta

- Le prestarían cien pesos

- Se han reunido en un área común con sus vecinos

- Es fácil reconocer a alguien que no es de la colonia o barrio, confía en los vecinos y confía en la policía.

La dimensión a nivel meso, que se refiere a la confianza estructural basada en las expectativas de vida, se midió seleccionando seis preguntas que representan los sentimientos de valoración del futuro de aspectos sociales (posibilidades de tener tiempo para convivir y divertirse; vivir y mantenerse seguro en su colonia o barrio y hogar; tener o mantener su propia casa o departamento) y económicos (posibilidad de crecer en su trabajo o profesión; tener un buen empleo y tener su propio negocio) para alcanzar una buena vida. Se parte del supuesto que las personas que tienen mayores expectativas 
sociales tendrían una mayor confianza estructural, lo que aumentaría los incentivos a actuar colectivamente en caso de una amenaza a su bienestar social. Mientras que las personas con mayores expectativas de vida centradas en su bienestar económico o individual compartirán menos valores colectivos, por lo tanto, ante una amenaza tenderán a enfrentarla de manera individual (Coleman, 1988).

Para evitar el problema de la endogeneidad o causalidad inversa que surge al realizar el análisis de los indicadores del capital social y la incidencia de las violencias, debido a que ciertas formas de violencia pueden influir en el capital social, por ejemplo, la participación puede disminuir si la violencia aumenta y provoca el temor de salir de casa, Lederman, Loayza y Menéndez (2002) sugieren el uso de variables instrumentales ficticias, agrupando las diferentes unidades de estudio por ubicación geográfica o nivel de desarrollo, asumiendo que esto homogeniza ciertos rasgos sociales que afectan el nivel de capital social. En este artículo no se emplearon directamente los índices correspondientes a cada jefe(a) del hogar sino un promedio de cada conglomerado del muestreo (UPM), para reflejar que el capital social es una construcción colectiva. Los conglomerados comprendían una media de 20 encuestados, con un mínimo de 7 y un máximo de 57.

Con el fin de lograr los objetivos planteados se realizaron dos diferentes tipos de análisis estadístico, el análisis de componentes principales (ACP) y una regresión lineal múltiple, en el programa estadístico SPSS v. 22. EI ACP permitió visualizar las relaciones entre el elevado número de variables que se tiene en las dos dimensiones empleadas del capital social, transformando el conjunto de variables originales en un nuevo conjunto de variables denominadas componentes principales (Fuentes, 2011), caracterizadas por estar correlacionadas entre sí y, además, ordenadas de acuerdo con la información que llevan incorporada.

Antes de aplicar el ACP se verificó que la correlación entre las variables analizadas fuera lo suficientemente grande como para justificar la factorización de la matriz de coeficientes de correlación. Esta comprobación se realizó mediante el índice de KaiserMeyer-Olkin (KMO), que parte de la hipótesis nula de que la matriz de coeficientes de correlación no es significativamente distinta de la matriz identidad. El índice KMO compara los valores de las correlaciones entre las variables y sus correlaciones parciales. Si el índice KMO está próximo a 1 , el ACP se puede hacer. Si el índice es bajo (próximo a 0), el ACP no será relevante. Además, con la finalidad de corroborar la relación o el modelo subyacente, en el ACP se rotaron los ejes, transformando la matriz factorial original en otra más simple que adecua mejor los ejes al aproximarlos a las variables correlacionadas, este procedimiento no altera la bondad de ajuste de la solución factorial y mantiene inalterados los porcentajes de varianza explicada que se distribuye entre los factores.

Para finalizar, se estimaron modelos de regresión lineal múltiple para cada uno de los componentes del capital social con el fin de determinar el efecto de la incidencia observacional de las diferentes formas de violencia. 


\section{Resultados}

En la dimensión redes sociales se extraen dos componentes principales denominados redes sociales primarias. El primero lo integraron las variables relacionadas con la extensión, grado de identificación, intensidad de la interacción social entre los familiares y conocidos que viven dentro de la colonia. El segundo es el de redes sociales organizadas que agrupa las variables sobre la participación en actividades comunes con sus vecinos y la membresía del/de la jefe(a) o la de otro miembro del hogar en alguna organización vecinal, tal y como se muestra en la Tabla 1 que contiene la matriz de cargas factoriales del análisis.

\section{Tabla 1 \\ Matriz de cargas factoriales de variables sobre redes sociales (matriz de componente rotado)}

\begin{tabular}{|l|c|c|}
\hline \multirow{2}{*}{ Redes sociales, KMO = $\mathbf{0 . 6 2}$} & \multicolumn{2}{c|}{ Componente } \\
\cline { 2 - 3 } & $\begin{array}{c}\text { Redes } \\
\text { primarias } \\
\text { organizadas }\end{array}$ \\
\hline Extensión de la red de familiares, amigos o conocidos & .651 & .760 \\
\hline Grado de identificación de los integrantes & .718 \\
\hline Intensidad de la interacción social & & \\
\hline Participación en actividades comunes con los vecinos & & .764 \\
\hline Membrecía en alguna organización vecinal & .778 \\
\hline \% de varianza & 30.81 & 25.60 \\
\hline
\end{tabular}

Fuente: Elaboración propia con base en la Ecopred, Inegi, 2014.

Método de extracción: análisis de componentes principales.

Con el grado de confianza interpersonal basada en la reciprocidad particular se calcularon tres componentes que responden a un orden de magnitud del valor material o simbólico del apoyo social que está relacionado con la intensidad de la confianza y la reciprocidad particular que existe entre los vecinos (Tabla 2). El primero de ellos denominado confianza interpersonal ausente, el cual está asociado a las variables sobre la confianza en los cuerpos policiales, independientemente de si han tenido un acercamiento con los mismos o no, y a una baja interacción y confianza en los vecinos.

El segundo denominado confianza interpersonal débil, el cual se centra en una confianza y un grado bajo de expectativas de ayuda entre vecinos, son los simples conocidos o conocidos de alguien más que se hacen favores de menor valor material y simbólico, por ejemplo, prestarse una herramienta.

El tercer componente es la confianza interpersonal fuerte, que refiere a un grado mayor de confianza y de expectativas de ayuda entre los vecinos, basada en las interacciones repetidas, así como en las experiencias previas de acompañamiento y 
reciprocidad, y con frecuencia tiene emociones intensas asociadas, por ejemplo, a dejar a los hijos(as) bajo el cuidado de un vecino(a).

Tabla 2

Matriz de cargas factoriales de las variables sobre la confianza interpersonal basada en la reciprocidad particular (matriz de componentes rotados)

\begin{tabular}{|l|c|c|c|}
\hline \multirow{2}{*}{$\begin{array}{l}\text { Grado de confianza interpersonal, } \\
\text { KMO }=\mathbf{0 . 8 3}\end{array}$} & $\begin{array}{c}|c| \\
\text { Componente } \\
\text { interpersonal } \\
\text { débil }\end{array}$ & $\begin{array}{c}\text { Confianza } \\
\text { interpersonal } \\
\text { fuerte }\end{array}$ & $\begin{array}{c}\text { Confianza } \\
\text { interpersonal } \\
\text { ausente }\end{array}$ \\
\hline $\begin{array}{l}\text { Los vecinos le cuidarían la casa si saliera } \\
\text { de la ciudad por varios días }\end{array}$ & .702 & & \\
\hline $\begin{array}{l}\text { Recurriría a sus vecinos si se encontrara } \\
\text { en un problema }\end{array}$ & .682 & & \\
\hline $\begin{array}{l}\text { Sus vecinos le prestarían una } \\
\text { herramienta }\end{array}$ & .783 & & \\
\hline $\begin{array}{l}\text { En caso de emergencia su vecino le } \\
\text { prestaría cien pesos }\end{array}$ & .614 & & \\
\hline $\begin{array}{l}\text { Confía en la gente de esta colonia o } \\
\text { barrio }\end{array}$ & .546 & & \\
\hline $\begin{array}{l}\text { Deja las llaves de la casa a sus vecinos } \\
\text { cuando sale de viaje }\end{array}$ & & .688 & \\
\hline $\begin{array}{l}\text { Han cuidado a sus hijos o a algún menor } \\
\text { bajo su responsabilidad sus vecinos }\end{array}$ & & .662 & \\
\hline $\begin{array}{l}\text { Se reúnen en un área común los } \\
\text { integrantes de su hogar y sus vecinos. }\end{array}$ & & .678 & \\
\hline $\begin{array}{l}\text { Es fácil reconocer a alguien que no es de } \\
\text { la colonia o barrio }\end{array}$ & & & .328 \\
\hline Confía en la policía en su colonia o barrio & & & \\
\hline$\%$ de varianza & 23.67 & 15.39 & \\
\hline
\end{tabular}

Fuente: Elaboración propia a partir de la Ecopred, Inegi, 2014.

Método de extracción: análisis de componentes principales.

La confianza estructural basada en las expectativas de vida futura está relacionada con los sentimientos de felicidad, eficacia personal y capacidad de influir en eventos amplios. Para esta dimensión se extraen dos componentes, las expectativas económicas referentes a la capacidad de ascender laboralmente y, por lo tanto, mejorar en las áreas económicas, y las expectativas sociales, que hace referencia a la convivencia familiar, la seguridad social y el tiempo de convivir y divertirse, que se refieren al bienestar social, cuyas cargas factoriales se muestran en la Tabla 3. 
Tabla 3

Matriz de cargas factoriales de las variables de la confianza estructural basada en las expectativas de vida futura (matriz de componentes rotados)

\begin{tabular}{|l|c|c|}
\hline \multirow{2}{*}{ Expectativas, KMO $=\mathbf{0 . 7 2}$} & \multicolumn{2}{|l|}{ Componente } \\
\cline { 2 - 3 } & $\begin{array}{l}\text { Expectativas } \\
\text { económicas }\end{array}$ & $\begin{array}{l}\text { Expectativas } \\
\text { sociales }\end{array}$ \\
\hline Crecerá en su trabajo o profesión & .912 & \\
\hline Tendrá un buen empleo & .903 & \\
\hline Tendrá su propio negocio o trabajo por su cuenta & .657 & .819 \\
\hline $\begin{array}{l}\text { Se sentirá y vivirá seguro en su colonia o barrio y } \\
\text { hogar }\end{array}$ & & .739 \\
\hline Tendrá tiempo para convivir y divertirse & & .649 \\
\hline $\begin{array}{l}\text { Tendrá o mantendrá su propia casa o departamento } \\
\text { (una propiedad) }\end{array}$ & & 29.08 \\
\hline \% de varianza & 37.09 & \\
\hline
\end{tabular}

Fuente: Elaboración propia a partir de la Ecopred, Inegi, 2014.

Método de extracción: análisis de componentes principales.

Para verificar la estabilidad de la estructura de los componentes que integran el capital social se realizó el análisis por separado para cada uno de los componentes y después para todos los componentes en conjunto, esperando obtener la misma estructura en ambos ejercicios. El análisis para las dimensiones separadas muestra una estructura en conjunto de seis componentes, explicando $62.3 \%$ de la varianza, con un KMO de 0.62 , los factores presentan un nivel de consistencia interna adecuado con un alfa de Cronbach superior a 0.8 .

Los resultados del análisis factorial muestran que las dos dimensiones del capital social (Tabla 4), redes sociales y confianza, se desglosan en seis componentes: redes primarias, redes organizadas, confianza interpersonal ausente, confianza interpersonal débil, confianza interpersonal fuerte, expectativas económicas y expectativas sociales que reflejan aspectos particulares del capital social. 
Tabla 4

Matriz de cargas factoriales del conjunto de las variables sobre capital social (matriz de componentes rotados)

\begin{tabular}{|c|c|c|c|c|c|c|}
\hline & \multicolumn{6}{|c|}{ Componente } \\
\hline & \multicolumn{2}{|c|}{ Redes } & \multicolumn{3}{|c|}{ Confianza } & \multirow{2}{*}{$\begin{array}{l}\text { Expectativas } \\
\text { sobre la vida }\end{array}$} \\
\hline & primarias & organizadas & débil & fuerte & ausente & \\
\hline $\begin{array}{l}\text { Extensión de la red de familiares, amigos o } \\
\text { conocidos }\end{array}$ & .542 & & & & & \\
\hline Grado de identificación de los integrantes & .636 & & & & & \\
\hline Intensidad de la interacción social & .595 & & & & & \\
\hline $\begin{array}{l}\text { Participación en actividades comunes con } \\
\text { los vecinos }\end{array}$ & & .760 & & & & \\
\hline Membresía en alguna organización vecinal & & .639 & & & & \\
\hline Los vecinos le cuidarían la casa & & & .706 & & & \\
\hline $\begin{array}{l}\text { Recurriría a sus vecinos si se encontrara en } \\
\text { un problema }\end{array}$ & & & .694 & & & \\
\hline Sus vecinos le prestarían una herramienta & & & .727 & & & \\
\hline $\begin{array}{l}\text { En caso de emergencia su vecino le } \\
\text { prestaría cien pesos }\end{array}$ & & & .587 & & & \\
\hline Confía en la gente de esta colonia o barrio & & & .601 & & & \\
\hline $\begin{array}{l}\text { Deja las llaves de la casa a sus vecinos } \\
\text { cuando salen de viaje }\end{array}$ & & & & .663 & & \\
\hline $\begin{array}{l}\text { Han cuidado a sus hijos o a algún menor } \\
\text { bajo su responsabilidad sus vecinos }\end{array}$ & & & & .705 & & \\
\hline $\begin{array}{l}\text { Se reúnen en un área común los integrantes } \\
\text { de su hogar y sus vecinos. }\end{array}$ & & & & .591 & & \\
\hline Confía en la policía en su colonia o barrio & & & & & .896 & \\
\hline Crecerá en su trabajo o profesión & & & & & & .844 \\
\hline Tendrá un buen empleo & & & & & & .843 \\
\hline $\begin{array}{l}\text { Tendrá su propio negocio o trabajo por su } \\
\text { cuenta }\end{array}$ & & & & & & .655 \\
\hline $\begin{array}{l}\text { Sentirá y vivirá seguro en su colonia o } \\
\text { barrio y hogar }\end{array}$ & & & & & & .574 \\
\hline Tendrá tiempo para convivir y divertirse & & & & & & .587 \\
\hline $\begin{array}{l}\text { Tendrá o mantendrá su propia casa o } \\
\text { departamento (una propiedad) }\end{array}$ & & & & & & .651 \\
\hline
\end{tabular}

Fuente: Elaboración propia a partir de la Ecopred, Inegi, 2014.

Método de extracción: análisis de componentes principales.

La estructura compuesta por seis componentes es más específica y refleja de forma más clara los tipos de redes sociales y grados de confianza interpersonal y estructural del capital social existentes entre los/las jefes(as) de hogar residentes de la ZMM. Estas variables obtenidas de los diferentes análisis serán utilizadas como posibles determinantes de la incidencia observacional de las violencias.

Análisis de componentes principales de la incidencia observada de las violencias

Para reducir el número de variables sobre los diferentes comportamientos delictivos y antisociales se realizó una tipología para aglutinar las diversas manifestaciones de las violencias percibidas por los/las jefes(as) de los hogares residentes en las colonias 
de la ZMM, empleando un análisis factorial de componentes principales, con rotación oblicua.

Se extraen cuatro componentes: el primero de ellos se denomina violencia entre pandillas, que agrupa a los delitos: grafiteando paredes o rayando autos, vendiendo piratería, vendiendo drogas, consumiendo drogas y peleando entre pandillas. El segundo componente es la violencia con arma de fuego, incluye disparando algún tipo de arma de fuego y prostituyéndose en la calle ${ }^{4}$. El tercer componente es la violencia contra el patrimonio, integra los delitos asaltando o robando casas, negocios o vehículos, asaltando o robando personas en la calle y amenazando o extorsionando. $Y$ el cuarto componente es la violencia por convivencia donde se considera realizar ruidos en tonos muy altos (por música, fiestas o realizando alguna actividad doméstica), ingiriendo alcohol en la vía pública y bloqueando las calles. La matriz de cargas factoriales se muestra en la Tabla 5:

Tabla 5

Matriz de las cargas factoriales de las violencias

\begin{tabular}{|c|c|c|c|c|}
\hline \multirow{2}{*}{$\begin{array}{l}\text { Violencias, KMO = } 0.877 \\
\text { En lo que va del año, qué tan frecuente ha } \\
\text { observado gente en su colonia o barrio... }\end{array}$} & \multicolumn{4}{|c|}{ Componente/Violencia asociada a... } \\
\hline & Pandillas & $\begin{array}{c}\text { Armas } \\
\text { de fuego }\end{array}$ & Patrimonio & Convivencia \\
\hline Haciendo ruido alto & & & & .729 \\
\hline Tomando alcohol en la calle & & & & .622 \\
\hline Bloqueando la calle & & & & .656 \\
\hline Grafiteando paredes o rayando autos & .640 & & & \\
\hline Vendiendo productos piratas & .538 & & & \\
\hline Vendiendo drogas & .604 & & & \\
\hline Consumiendo drogas & .645 & & & \\
\hline Peleando entre pandillas & .522 & & & \\
\hline Prostituyéndose & & .628 & & \\
\hline Disparando algún tipo de arma de fuego & & .639 & & \\
\hline Asaltando o robando casas, negocios o vehículos. & & & .783 & \\
\hline Asaltando o robando a personas en la calle & & & .804 & \\
\hline Amenazando o extorsionando & & & .598 & \\
\hline \% de varianza & 15.6 & 12.9 & 12.6 & 11.3 \\
\hline
\end{tabular}

Fuente: Elaboración propia a partir de la Ecopred, Inegi, 2014.

Nota: Rompiendo ventanas de casas, negocios o autos, u otros objetos; Discutiendo o peleando entre vecinos y Jugando arrancones tuvieron cargas factoriales $<0.5$.

\footnotetext{
4 Llama la atención que se unifique en un mismo delito, prostitución y disparando armas de fuego, esto posiblemente se deba a que en las colonias o barrios donde hay una mayor incidencia observacional de personas prostituyéndose en las calles, se tenga la percepción de que son inseguras, por lo que también exista una mayor incidencia de personas disparando armas de fuego, aunque se tendría que examinar con mayor profundidad cuáles son los elementos que los unifican en una misma categoría.
} 
Modelo de regresión lineal múltiple para los componentes de las dimensiones del capital social y la percepción de la violencia

Los resultados del modelo de regresión lineal múltiple muestran el efecto que tienen los componentes del capital social en la incidencia observacional de las violencias causadas por pandillas, por arma de fuego, contra el patrimonio, por convivencia. Se observa que el componente redes primarias fue estadísticamente significativo en la incidencia observacional de la violencia entre pandillas y por la convivencia (Tabla 6). Mientras que, en la violencia entre pandillas, las redes primarias mostraron un coeficiente de signo negativo, es decir a medida que aumenta la extensión, grado de identificación e intensidad de la interacción social entre los familiares y conocidos que viven dentro de la colonia o barrio disminuye la presencia de las violencias relacionadas con el grafiti, venta de piratería, venta y consumo de drogas y peleas entre pandillas.

Sin embargo, las redes primarias mostraron un coeficiente con signo positivo en la incidencia observacional de la violencia por convivencia, es decir, mientras aumentan las interacciones entre los familiares, conocidos y amigos se incrementa la presencia de conductas antisociales como hacer ruidos en tono muy alto (por música, fiestas o actividades domésticas), bloquear las calles o ingerir alcohol en la vía pública. Esto posiblemente se debe a que las personas se sienten en confianza con sus vecinos por lo que trasladan ciertas actividades privadas al espacio público. 
Tabla 6

Efecto de los componentes del capital social según la incidencia observacional de la violencia

\begin{tabular}{|c|c|c|c|c|}
\hline & $\begin{array}{l}\text { Violencia } \\
\text { entre } \\
\text { pandillas }\end{array}$ & $\begin{array}{l}\text { Violencia por } \\
\text { arma de fuego }\end{array}$ & $\begin{array}{l}\text { Violencia } \\
\text { contra el } \\
\text { patrimonio }\end{array}$ & $\begin{array}{l}\text { Violencia por } \\
\text { la convivencia }\end{array}$ \\
\hline (Constante) & 0.025 & 0.016 & 0.024 & -0.058 \\
\hline ACP Redes primarias & $-0.087 * *$ & 0.005 & -0.055 & $0.003 * *$ \\
\hline ACP Redes organizadas & $-0.041 * *$ & -0.051 & -0.020 & -0.026 \\
\hline ACP Confianza ausente & $0.106^{* *}$ & $0.069 * *$ & $0.081 * *$ & $0.140 * *$ \\
\hline ACP Confianza débil & $0.125^{* *}$ & $0.072 * *$ & $0.084 * *$ & $0.071 * *$ \\
\hline ACP Confianza fuerte & $-0.076 * *$ & -0.035 & -0.017 & $-0.122 * *$ \\
\hline $\begin{array}{l}\text { ACP Expectativas } \\
\text { económicas }\end{array}$ & $0.078 *$ & $0.036 *$ & $0.066 * *$ & $0.123 * *$ \\
\hline $\begin{array}{l}\text { ACP Expectativas } \\
\text { sociales }\end{array}$ & $-0.167 * *$ & -0.008 & $-0.048 * *$ & -0.053 \\
\hline ACPG Redes primarias & $-0.063 * *$ & -0.005 & -0.018 & $0.065 * *$ \\
\hline ACPG Redes organizadas & $-0.038^{*}$ & -0.035 & 0.014 & -0.038 \\
\hline ACPG Confianza ausente & $0.198 * *$ & $0.082 * *$ & $0.142 * *$ & $0.118 * *$ \\
\hline ACPG Confianza débil & $0.111^{* *}$ & $0.080 * *$ & $0.093 * *$ & 0.042 \\
\hline ACPG Confianza fuerte & $-0.105^{* *}$ & -0.036 & -0.042 & $-0.098 * *$ \\
\hline $\begin{array}{l}\text { ACPG Expectativas } \\
\text { económicas y sociales }\end{array}$ & $-0.039 *$ & -0.014 & -0.045 & $-0.116 * *$ \\
\hline $\mathrm{R}$ & 0.542 & 0.311 & 0.376 & 0.578 \\
\hline $\mathrm{R}$ cuadrado & .359 & .239 & .190 & .461 \\
\hline R cuadrado ajustado & .326 & .132 & .126 & .345 \\
\hline EEE & 1.03 & 0.97 & 1.02 & 1.00 \\
\hline Durbin-Watson & 1.695 & 1.548 & 1.827 & 1.667 \\
\hline $\mathrm{N}$ & 843 & 781 & 1273 & 1213 \\
\hline
\end{tabular}

Fuente: Elaboración propia a partir de la Ecopred, Inegi, 2014.

$* \mathrm{p}<.05 ; * * \mathrm{p}<.01 ; \mathrm{p}<.001$.

El componente redes organizadas en la colonia solo fue significativo en la violencia entre pandillas, mostrando un coeficiente de signo negativo. Esto comprueba que una mayor presencia de asociativismo es capaz de disminuir la percepción de violencia por venta y consumo de drogas y peleas entre pandillas en las colonias o barrios.

Es importante señalar que los componentes de confianza interpersonal ausente y débil fueron estadísticamente significativos para los cuatro tipos de violencia, en todos los 
casos, el signo fue positivo. Si la confianza y reciprocidad particular es nula o débil entre los vecinos, aumenta la incidencia observacional de todas las violencias (entre pandillas, por armas de fuego, contra el patrimonio y por convivencia). Es decir, entre más deteriorada se encuentre la confianza interpersonal basada en la reciprocidad entre los vecinos, mayor es la presencia de todas las violencias y comportamientos antisociales en el entorno de su comunidad.

El componente confianza interpersonal fuerte fue significativo para la violencia entre pandillas y por convivencia, en ambos casos, el signo del coeficiente fue negativo. Cuando existe confianza interpersonal fuerte basada en altas expectativas de ayuda entre los vecinos es significativa la disminución de la incidencia de la violencia entre pandillas y por convivencia en su entorno. Sin embargo, la confianza interpersonal fuerte no mostró un efecto significativo en las violencias contra el patrimonio y por armas de fuego.

El componente que mide las expectativas económicas fue significativo para los cuatro tipos de violencia: entre pandillas, por armas de fuego, contra el patrimonio y por convivencia, y todos los coeficientes presentaron un signo positivo en cuyo caso se interpreta que cuando se tienen mayores expectativas económicas, basadas en la capacidad de ascender laboralmente $y$, por lo tanto, mejorar en las áreas económicas contribuye al aumento de la incidencia de todas las violencias en el entorno que habitan.

Las altas expectativas de vida social fueron significativas en las violencias entre pandillas y contra el patrimonio, el signo que presentó fue negativo, lo que significa que cuando las personas tienen como prioridad la seguridad y la convivencia familiar estos tipos de delito disminuyen, posiblemente porque existe un mayor involucramiento de las personas para realizar acciones colectivas para contener este tipo de violencias.

El resultado global de los componentes principales en las dimensiones del capital social en relación con los cuatro tipos de violencia mostró que casi todos los coeficientes mantienen el mismo nivel de significancia, con excepción de las redes organizadas y la violencia entre pandillas, tendencia que concuerda con la registrada en los resultados de los componentes principales particulares de las dimensiones del capital social por lo que se corroboró la estabilidad de los resultados.

\section{Discusión}

El presente artículo tuvo como objetivo analizar la relación entre algunas de las dimensiones constitutivas del capital social y la incidencia observacional de diferentes tipos de violencias en la Zona Metropolitana de Monterrey. A partir de los resultados se corroboró que el capital social y la violencia no tienen una relación univoca como ha sido planteado en varios estudios (Glaeser, Sacerdote y Scheinkman, 1996; Sampson, Raudenbush y Earls, 1997; Rosenfeld, Messner y Baumer, 2001; Rosero-Bixby, 2006; Núñez, 2010; López-Rodríguez, Soloaga y De La Torre, 2014; Jara, 2015). 
Indagando en las relaciones particulares entre los diferentes componentes del capital social con los diversos tipos de violencia se encontró que cada componente del capital social produce efectos diferentes sobre cada tipo de violencia, comprobando así la siguiente hipótesis: los componentes del capital social muestran efectos diferenciales sobre la presencia de las violencias, por lo que la relación entre los componentes del capital social y la incidencia observacional de las distintas violencias varían.

En particular, las redes sociales primarias y organizadas, la confianza interpersonal fuerte y las altas expectativas de vida social fueron los componentes del capital social que tuvieron un efecto positivo en la disminución de tres tipos de violencias: por convivencia, entre pandillas y contra el patrimonio. La existencia de un capital social fuerte disminuye la presencia de los delitos menores.

Estos resultados concuerdan con las investigaciones de Glaeser, Sacerdote y Scheinkman (1996), Núñez (2010), López-Rodríguez, Soloaga y De La Torre (2014) y Jara (2015), quienes encontraron como la frecuencia de las interacciones, la participación ciudadana, el capital social y la asociatividad tienden a crecer cuando los delitos son menores.

La confianza interpersonal basada en la reciprocidad particular ausente o débil y las altas expectativas de vida centradas en lo económico aumentaron la incidencia de todos los tipos de violencia: por convivencia, entre pandillas, contra el patrimonio y por arma de fuego. Es decir, un capital social débil entre los vecinos aumenta la sensación de peligro y la violencia en el entorno que habitan, o bien, la violencia provoca comportamientos de aislamiento social que impiden la convivencia social y la formación de capital social.

Los resultados de este artículo muestran también como la mera existencia de capital social entre las personas que cohabitan en un entorno urbano no permite contener los efectos de todas las violencias presentes en la trama social. El capital social como una estrategia para contener los impactos de las violencias tiene límites, ninguna de las dos dimensiones, las redes sociales y la confianza, parece inhibir los delitos cometidos con arma de fuego, homicidios, prostitución y violaciones, es decir, delitos de alto impacto.

Se confirma que la confianza interpersonal basada en la reciprocidad particular se encuentra erosionada en espacios urbanos en donde hay alta presencia del narcotráfico, el consumo de drogas y la violencia (Lunecke y Ruiz, 2006). Por el contrario, en donde los delitos son menores, el capital social y la asociatividad tienden a ser más fuertes y a tener más presencia.

La estrategia de movilizar el capital social para enfrentar las violencias en la ZMM debería centrarse en fortalecer las redes sociales de los grupos organizados, la confianza interpersonal basada en la reciprocidad y fomentar altas expectativas sociales de la vida entre los vecinos para aumentar la protección de su entorno, disminuir los riesgos entre los miembros de la comunidad y favorecer la resolución pacífica de conflictos al interior de las comunidades. Esta estrategia solo será efectiva si las instituciones y organismos encargados de la seguridad apoyan y fortalecen el desarrollo del capital social. 
La principal limitación de este artículo es la disponibilidad de información empírica, pues la Ecopred no cuenta con información que se ajuste por completo a los enfoques teóricos para medir el capital social (Bourdieu, 1988; Coleman, 1988, 1990; Grootaert et al., 2004), por lo que resulta necesario realizar un esfuerzo sistemático de recabar información empírica sobre los procesos que fortalecen o deterioran el capital social en contextos de violencia.

\section{Referências bibliográficas}

ARRIAGADA, I. "Capital social: potencialidades y limitaciones analíticas de un concepto". Estudios Sociológicos, México, vol. XXI, no 3, p. 557-584, 2003.

BellanCA, J.; BRANDT, R. (eds.). 21st Century Skills: rethinking how students learn. Bloomington: Solution Tree Press, 2010.

BOURDIEU, P. "The social space and the genesis of groups". Theory and Society, New York, USA, vol. 14, n० 6, JSTOR Essential Collection, p. 723-744, 1985.

BouRdieU, P.; WACQUANT, L. An invitation to reflexive sociology. Chicago, USA: University of Chicago Press, 1992.

CARREÓn, F. "De la violencia urbana a la convivencia ciudadana". Ciudad y Violencias en América Latina, Quito, Ecuador, vol. 14, nº 2, Programa de Gestión Urbana, p. 5-22, 1994.

Coleman, J. "Social capital in the creation of human capital". American Journal of Sociology, Chicago, USA, no 94, University of Chicago Press, p. 95-120, 1988.

. Foundations of social theory. Cambridge, USA: Belknap Press, 1990.

CóRDova, M. "Percepción de inseguridad: una aproximación transversal". Ciudad Segura, vol. 15, no 1, Programa Estudios de la Ciudad, Ecuador, Flacso, p. 4-11, 2007.

CRUZ, C. E.; ContreRAS-IbáÑEZ, C. Confianza, normas de reciprocidad y redes de compromiso cívico: una propuesta de evaluación comunitaria del capital social. In: CHARRY, C. I.; CONTRERAS-IBáÑEZ, C. (coords.). Capital social: enfoques alternativos, México: Editorial Siglo XXI, 2015.

CRUZ, J. M. "La victimización por violencia urbana: niveles y factores asociados en ciudades de América Latina y España". Revista Panamericana de la Salud Pública, Washington, USA, vol. 5, no 4, Organización Panamericana de la Salud, p. 256-267, 1999.

DAMmeRT, L.; ZUÑIGA L. Seguridad y violencia: desafíos para la ciudadanía. Santiago, Chile: Facultad Latinoamericana de Ciencias Sociales, 2007.

Del Olmo, R. "Ciudades duras y violencia urbana". Nueva sociedad, Buenos Aires, Argentina, no 167, Fundación Nueva Sociedad, p. 1-15, 2000.

DuRkHeim, E. Las reglas del método sociológico. Buenos Aires: La pléyade, 1985.

DURSTON, J. ¿Qué es el capital social comunitario?. Santiago de Chile: Cepal, División de Desarrollo Social, 2000.

FAIST, T. "Towards a political sociology of transnationalism". European Journal of Sociology, Cambridge, USA, vol. 45, no 3, Cambridge University Press, p. 19-54, 2004. 
Foster, G. "The dyadic contract: a model for the social structure of a mexican peasant village". American Anthropologist, vol. 63, $\mathrm{n}^{\circ}$ 6, p. 1.173-1.192, 1961.

FUENTES, S. Componentes principales. Facultad de ciencias económicas y empresariales, Ciudad de México, México: Universidad Autónoma Metropolitana, 2011.

Glaeser, E.; SACERdote, B.; Scheinkman, J. "Crime and social interactions". Quarterly Journal of Economics, Oxford, UK, no CXI, University of Oxford Press, p. 507-548, 1996.

GranovetTer, M. "The strength of weak ties". American Journal of Sociology, vol. 78, no 6, p. 1.360$1.380,1973$.

GRoOtAeRT, C., et al. "Measuring social capital: an integrated questionnaire". World Bank Working Paper, Washington, USA, nº 18, World Bank, p. 1-51, 2004.

HiRsCHMAN, A. O. Getting ahead collectively: grassroots experiences in Latin America. Oxford: Pergamon Press, 1984.

INEGI. Encuesta de Cohesión Social para la Prevención de la Violencia y la Delincuencia (Ecopred). Aguascalientes, México, 2014.

JARA, A. "Capital social y delitos violentos. Análisis para 101 comunas urbanas en Chile". Revista Estudios de Políticas Públicas, Santiago, Chile, no 1, Universidad Autónoma de Chile, p. 193-212, 2015.

LATORRE, M. C. Sobre la relación positiva entre el capital social y la violencia urbana: un análisis teórico y empírico. Documentos Cede. Colombia: Universidad de los Andes Cede, 2004.

Lederman, D.; LoAyzA, N.; MenÉndez, A. M. "Violent crime: does social capital matter?". Economic Development and Cultural Change, Washington, USA, nº 50, World Bank, p. 509-539, 2002.

LIN, N. Social capital: A theory of social structure and action. Cambridge, USA: Cambridge University Press, 2001.

Lomnitz, L. ¿Cómo sobreviven los marginados?. México, DF: Siglo XXI, 1978.

López-Rodríguez, P.; Soloaga, I.; De La TorRe, R. "El efecto de la percepción de la violencia en el capital social en México". Bienestar y Política Social, México, vol. 9, no 1, Conferencia Interamericana de Seguridad Social, p. 79-110, 2014.

LUHmAnN, N. Confianza. Barcelona: Universidad Iberoamericana-Anthropos, 1996.

LUNECKE, A.; RUIZ, J. C. Barrios urbanos críticos en materia de violencia y delincuencia: marco de análisis para la construcción de indicadores. Chile: Universidad Alberto Hurtado, 2006.

NARAYAN, D., PRITCHETT, L. "Cents and sociability: household income and social capital in rural tanzania". Economic Development and Cultural Change, vol. 47, n 4, p. 871-897, 1999.

NúÑEZ, N. "Relación entre capital social y seguridad ciudadana en la comuna de La Pintana". Tesis de la Universidad de Chile, Santiago, Chile, 2010.

OSTROM, E.; AHN, T. K. "Una perspectiva del capital social desde las ciencias sociales: capital social y acción colectiva". Revista Mexicana de Sociología, México, vol. 65, no 1, p. 155-233, 2003.

PoRTela, M.; Neira. I. "Capital Social y bienestar subjetivo. Un análisis para España considerando sus regiones". Investigaciones Regionales, Santiago de Compostela, España, no 23, Universidad Santiago de Compostela, p. 5-27, 2012. 
PORTES, A. Economic sociology and the sociology of immigration: A conceptual overview. In: PORTES, A. (ed.). The economic sociology of immigration. New York, USA: Russell Sage Foundation, 1995.

. "Social capital: its origins and applications in modern sociology". Annual Review of Sociology, New Jersey, USA, no 24, Pricenton University Press, p. 1-24, 1998.

PUTNAM, R. Making democracy work: civic traditions in modern Italy. New Jersey, USA: Princeton University Press, 1993.

Rosenfeld, R.; Messner, S.; Baumer, E. "Social capital and homicide". Social Forces, Oxford, UK, vol. 80, n० 1, University of Oxford Press, p. 283-310, 2001.

Rosero-BIXBY, L. "Capital social, asentamientos urbanos y comportamiento demográfico en América Latina". Notas de Población, Toluca, México, no 81, Universidad Autónoma del Estado de México, p. 73-98, 2006.

SAFA, P.; RAmírez, J. "Deterioro urbano y calidad de vida en las grandes urbes: la participación de las mujeres en las organizaciones vecinales". La Ventana, México, no 34, Universidad Autónoma de Guadalajara, p. 110-144, 2011.

SAMPSON, R.; RAUDENBUSH, S.; EARLS, F. "Neighborhoods and violent crime: a multilevel study of collective efficacy". Science, USA, vol. 277, no 5.328, American Association for the Advancement of Science, p. 918-24, 1997.

SeSNSP - Secretariado Ejecutivo del Sistema Nacional de Seguridad Pública. "Reporte de incidencia delictiva del fuero común, modificaciones a las estadísticas por entidad federativas". México: Procuradurías de Justicia y Fiscalías Federales, 2017. Disponible en:

<http://secretariadoejecutivo.gob.mx/incidencia-delictiva/incidencia-delictiva-fuero-comun.php>. Acceso en: 12 jun. 2017.

Tedesco, L. Violencia urbana: un desafío al fortalecimiento institucional. El caso de América Latina. Madrid, España: Fundación para las Relaciones Internacionales y el Dialogo Exterior, 2009.

\section{Resumo}

Percepções de violência e capital social em Nuevo León, México

O objetivo deste artigo é analisar a relação entre algumas dimensões do capital social e a incidência observacional de diferentes tipos de violência na Região Metropolitana de Monterrey. Nesta análise utilizamos como fonte primária de informação a base de dados da Encuesta de Cohesión Social para la Prevención de la Violencia y la Delincuencia (Ecopred). Os dados da pesquisa foram analisados utilizando a técnica estatística dos componentes principais para determinar a tipologia do capital social e os diferentes tipos de violência. Posteriormente, foi desenvolvido um modelo de regressão linear múltipla. Com base nos resultados, mostrou-se que três dimensões do capital social - redes sociais organizadas, forte confiança interpessoal e altas expectativas sociais - tiveram um efeito positivo na redução da violência por coabitação, gangues, propriedade e crimes menores. Em conclusão, ter alguma dimensão de capital social é uma estratégia da comunidade para conter a violência.

Palavras-chave: capital social; confiança; violência; crimes; Nuevo León

\section{Abstract}

Perceptions of violence and social capital in Nuevo León, Mexico

The objective of this study is to analyze the relationship between some dimensions of social capital and the observational incidence of different types of violence in the Metropolitan Area of Monterrey. In this analysis, we use the database of the Social Cohesion Survey for the Prevention of Violence and 
Delinquency (ECOPRED) as the primary source of information. The research data were analyzed using the statistical technique of the main components to determine the typology of social capital and the different types of violence. Subsequently, a multiple linear regression model was developed. Based on the results, it was shown that three dimensions of social capital, organized social networks, strong interpersonal trust, and high social expectations had a positive effect on the reduction of violence due to cohabitation or gangs, of damages against property, and of minor crimes. In conclusion, having some dimension of social capital is a community strategy to contain violence.

Keywords: social capital; trust; violence; crime; Nuevo Leon

\section{Résumé}

Perceptions de la violence et du capital social à Nuevo León, au Mexique

L'objectif de cette étude est d'analyser la relation entre certaines dimensions du capital social et l'incidence d'observation de différents types de violence dans la région métropolitaine de Monterrey. Dans cette analyse, notre source principale d'information est la base de données de I'Enquête de cohésion sociale pour la prévention de la violence et de la délinquance (Ecopred). Les données de la recherche ont été analysées en utilisant la technique statistique des principaux composants pour déterminer la typologie du capital social et les différents types de violence. Ultérieurement, un modèle de régression linéaire multiple a été développé. Les résultats ont montré que trois dimensions du capital social, des réseaux sociaux organisés, une confiance interpersonnelle forte et des attentes sociales élevées avaient un effet positif sur la réduction de la violence due à la cohabitation, aux gangs, aux biens et aux délits mineurs. En conclusion, avoir une certaine dimension du capital social est une stratégie communautaire visant à contenir la violence.

Mots-clés: capital social; confiance; violence; crimes; Nuevo León

Artigo submetido à publicação em 31 de agosto de 2018 . Versão final aprovada em 20 de maio de 2019.

Opinião Pública adota a licença Creative Commons CC-BY. 